\title{
Two-time intensity correlation in a driven three-level system
}

\author{
H. Huang, S.-Y. Zhu, and M. S. Zubairy* \\ Department of Physics, Hong Kong Baptist University, Hong Kong \\ M. O. Scully \\ Department of Physics, Texas A \& M University, College Station, Texas 77843 \\ and Max-Planck-Institut für Quantenoptik, D-85748 Garching, Germany
}

(Received 22 August 1995)

\begin{abstract}
We examine the various two-time fluorescence intensity correlations emitted by a driven three-level atom in a cascade configuration. For a strongly driven atom, the two intensity autocorrelation functions, one from the upper transition, the other from the lower transition, exhibit different behaviors. In addition to the an oscillation similar to the two-level intensity correlation we find an additional oscillation for the upper transition only. This is shown to be due to the spontaneous decay between the lower transition levels.

PACS number(s): 42.50.Lc, 42.50.Ct
\end{abstract}

\section{INTRODUCTION}

The driven three-level system has been studied extensively in connection with a variety of phenomena associated with quantum coherence such as quantum beat, coherent trapping, and laser without inversion [1-3]. When the atom is driven by two coherence sources, the two driving fields create splits in the dressed state picture, and thus change the characteristics of resonance fluorescence. Such a change in the resonance fluorescence spectrum has been studied by Narducci and co-workers [4-6]. As each level is split into several sublevels, the transition from one to another has several possible paths [7]. This was the picture used in a recent analysis to treat the spontaneous photon emission spectrum from a level that is split due to the strong coupling to another level [8]. The results there, such as a dark line in the spectrum, also apply to the case where one can no longer use the picture of a superposition of two pure-state components.

For two-photon processes, there are more avenues for quantum interference and manifestation of quantum interference is also expected in the two-photon measurement signals. Such features in the two-level fluorescence intensity correlation have been known since the early years of quantum optics [9]. A simple analytic result is available. The twolevel atom fluorescence problem has been under continued study, focused primarily on the effects by introducing squeezed driving fields [10]. On the other hand, studies on three-level systems have been relatively few. The three-level atom when driven by two fields is expected to exhibit new features as the two driving fields acting jointly on the atom will inevitably produce signatures that cannot be attributed to a two-level nature. We present here our study on the fluorescence intensity correlation of the driven three-level atom. We start with a discussion of the three-level atom, the atomic master equation, and the use of the quantum regression theorem to derive the two-photon intensity correlations using the solution of the master equation. The numerical method and

\footnotetext{
*Permanent address: Department of Electronics, Quaid-i-Azam University, Islamabad, Pakistan.
}

results for these correlation functions are discussed in the next section. Of particular interest is the comparison between the upper fluorescence correlation and its counterpart for the lower transition. In the two-level model, such correlation depends only on the lifetime of the upper level involved, and the Rabi frequency between the two levels. Thus in the two cases where either the upper transition or the lower transition alone is driven, we expect the corresponding fluorescence intensity correlations to exhibit the same properties for similar parameters. What will happen in the situation when both transitions are driven with the same Rabi frequency? Intuition would suggest identical features in both intensity selfcorrelation functions. Our numerical result, however, shows that the two do differ. The cause of this counterintuitive difference is only revealed in the following discussion, as we cannot truly isolate the two levels involved in the fluorescence photon emission; the third level is linked to the former by both the other driving field as well as the vacuum modes (spontaneous emission). The result is an additional oscillation component at one-half the familiar two-level Rabi oscillation frequency, present only in the upper fluorescence signal.

\section{DRIVEN THREE-LEVEL SYSTEM}

We consider the three-level system as illustrated in Fig. 1. Two laser beams couple the levels $|1\rangle$ and $|2\rangle$ with the Rabi frequency $\Omega_{1}$, and $|2\rangle$ and $|3\rangle$ with the Rabi frequency $\Omega_{2}$. We assume that the two lasers are both on resonance, and the only dissipative terms are due to the spontaneous decays of the levels $|1\rangle$ and $|2\rangle$.

The intensity correlation measurement performed on the two fluorescence signals $I_{1}$ and $I_{2}$ can be expressed in terms of the atomic operators. In general we can write

$$
\begin{aligned}
\left\langle: I_{i}(t) I_{j}(t+\tau):\right\rangle= & \left\langle(|i\rangle\langle i+1|)_{t}(|j\rangle\langle j+1|)_{t+r}\right. \\
& \left.\times(|j+1\rangle\langle j|)_{t+r}(|i+1\rangle\langle i|)_{t}\right\rangle \\
= & \left\langle(|i\rangle\langle i+1|)_{t}(|j\rangle\langle j|)_{t+r}(|i+1\rangle\langle i|)_{t}\right\rangle,
\end{aligned}
$$


where $i, j=1,2$.

By the quantum regression theorem [11], for any three operators $Q_{\alpha}, Q_{\beta}, Q_{\gamma}$, the two-time product $\left\langle Q_{\alpha}(t) Q_{\beta}(t+\tau) Q_{\gamma}(t)\right\rangle$ can be expressed as

$\left\langle Q_{\alpha}(t) Q_{\beta}(t+\tau) Q_{\gamma}(t)\right\rangle$

$$
=\left\langle Q_{\alpha}(t) \sum_{\beta^{\prime}} h_{\beta \beta^{\prime}}(\tau) Q_{\beta^{\prime}}(t) Q_{\gamma}(t)\right\rangle .
$$

Here the $c$-number coefficients $h_{\alpha \beta}$ are derived from the solution of the Heisenberg equation for all operators, $Q_{\alpha}(t)=\Sigma_{\beta} h_{\alpha \beta}(t) Q_{\beta}(0)$. The right-hand side (RHS) of Eq. (1) can then be written as

$$
\begin{gathered}
\left\langle(|i\rangle\langle i+1|)_{t} \sum_{k l} h_{j j ; k l}(\tau)(|k\rangle\langle l|)_{t}(|i+1\rangle\langle j|)_{t}\right\rangle \\
=h_{j j ; i+1, i+1}(\tau)\left\langle(|i\rangle\langle i|)_{t}\right\rangle .
\end{gathered}
$$

Therefore the intensity correlations can be expressed in terms of the steady-state populations and the evolution operator for the density matrix:

$$
G_{i j}(\tau)=\frac{\left\langle: I_{i}(t) I_{j}(t+\tau):\right\rangle}{\langle\mid i\rangle\langle i \mid\rangle\langle\mid j\rangle\langle j \mid\rangle}=\frac{P_{i+1 \rightarrow j}(\tau)}{P_{j}},
$$

where $P_{j}$ is the steady-state population in $|j\rangle$, and $P_{i+1 \rightarrow j}(\tau)$ is the probability of population transfer from $|i+1\rangle$, the final state of the first emission, to level $|j\rangle$, the initial state of the second emission, in the time interval $\tau$. To find this probability we go to the atomic master equation [12]

$$
\begin{gathered}
\dot{\rho}_{11}=-2 \gamma_{1} \rho_{11}-i\left(\Omega_{1} \rho_{21}-\Omega_{1} \rho_{12}\right), \\
\dot{\rho}_{12}=-\left(\gamma_{1}+\gamma_{2}\right) \rho_{12}-i\left(\Omega_{1} \rho_{22}-\Omega_{1} \rho_{11}-\Omega_{2} \rho_{13}\right), \\
\dot{\rho}_{13}=-\gamma_{1} \rho_{13}-i\left(\Omega_{1} \rho_{23}-\Omega_{2} \rho_{12}\right), \\
\dot{\rho}_{21}=-\left(\gamma_{1}+\gamma_{2}\right) \rho_{21}-i\left(\Omega_{1} \rho_{11}+\Omega_{1} \rho_{31}-\Omega_{1} \rho_{22}\right), \\
\dot{\rho}_{22}=2 \gamma_{1} \rho_{11}-2 \gamma_{2} \rho_{22}-i\left(\Omega_{1} \rho_{12}+\Omega_{2} \rho_{32}-\Omega_{1} \rho_{21}-\Omega_{2} \rho_{23}\right), \\
\dot{\rho}_{23}=-\gamma_{2} \rho_{23}-i\left(\Omega_{1} \rho_{13}+\Omega_{2} \rho_{33}-\Omega_{2} \rho_{22}\right), \\
\dot{\rho}_{31}=-\gamma_{1} \rho_{31}-i\left(\Omega_{2} \rho_{21}-\Omega_{1} \rho_{32}\right), \\
\dot{\rho}_{32}=-\gamma_{2} \rho_{32}-i\left(\Omega_{2} \rho_{22}-\Omega_{1} \rho_{31}-\Omega_{2} \rho_{33}\right), \\
\dot{\rho}_{33}=2 \gamma_{2} \rho_{22}-i\left(\Omega_{2} \rho_{23}-\Omega_{2} \rho_{32}\right) .
\end{gathered}
$$

Here for simplicity we have assumed the lasers to be on resonance and the Rabi frequencies $\Omega_{1}, \Omega_{2}$ to be real. The average population $P_{i}$ is obtained directly from the steadystate solution of Eq. (5). The transition probability $P_{i \rightarrow j}(\tau)=h_{j j ; i i}$ is the solution for the density matrix component $\rho_{j j}(\tau)$ with the initial condition $\rho_{i i}=1$. The four different intensity correlations are thus

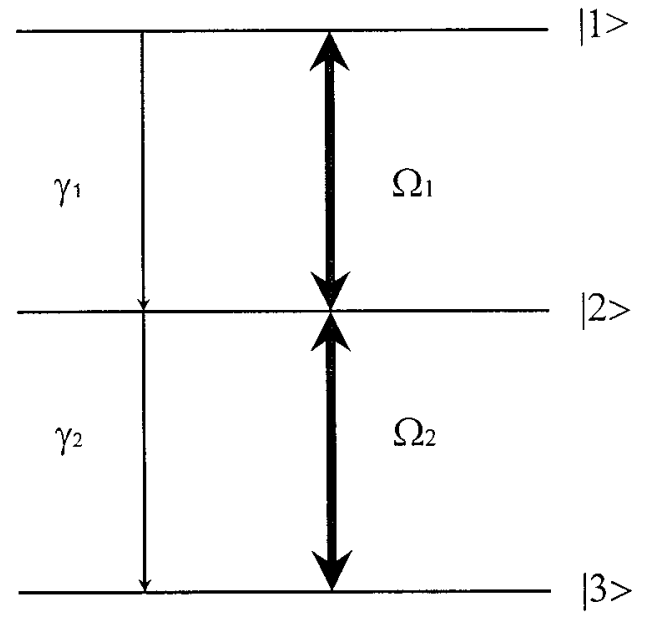

FIG. 1. A three-level atom resonantly driven by two lasers.

$$
\begin{aligned}
& G_{1,1}(\tau)=\frac{P_{2 \rightarrow 1}(\tau)}{P_{1}}, \\
& G_{2,2}(\tau)=\frac{P_{3 \rightarrow 2}(\tau)}{P_{2}}, \\
& G_{1,2}(\tau)=\frac{P_{2 \rightarrow 2}(\tau)}{P_{2}}, \\
& G_{2,1}(\tau)=\frac{P_{3 \rightarrow 1}(\tau)}{P_{1}} .
\end{aligned}
$$

\section{SOLUTION OF ATOMIC DENSITY MATRIX}

Formally we can write the nine elements of the density matrix $\rho$ as a nine-component array $\phi$, with

$$
\begin{gathered}
\phi_{1}=\rho_{11}, \quad \phi_{2}=\rho_{12}, \quad \phi_{3}=\rho_{13}, \ldots, \\
\phi_{8}=\rho_{32}, \quad \phi_{9}=\rho_{33} .
\end{gathered}
$$

and Eq. (5) can be written as

$$
\dot{\phi}=\mathbf{A} \phi
$$

where $\mathbf{A}$ is a $9 \times 9$ matrix containing the coefficients on the RHS of Eq. (5). Thus Eq. (6) becomes

$$
\begin{aligned}
& G_{1,1}(\tau)=\frac{\left(e^{\mathbf{A} \tau}\right)_{15}}{P_{1}}, \\
& G_{2,2}(\tau)=\frac{\left(e^{\mathbf{A} \tau}\right)_{59}}{P_{2}}, \\
& G_{1,2}(\tau)=\frac{\left(e^{\mathbf{A} \tau}\right)_{55}}{P_{2}}, \\
& G_{2,1}(\tau)=\frac{\left(e^{\mathbf{A} \tau}\right)_{19}}{P_{1}} .
\end{aligned}
$$


The general formalism as given above, however, does not allow an analytical solution. As the atomic density matrix no longer represents a pure state, it is difficult to identify terms contributing to interference effects except for several limiting cases.

In the limit when the driving fields are weak, the intensity autocorrelations each resembles the corresponding two-level case, but none possesses interesting features. This can be viewed in two ways. First, the weak fields do not induce sufficiently large level splits resolvable beyond the limit posed by the linewidth. Second, the decays occur before the impact of the driving fields can be felt. Therefore the process of an atom decaying from level $|1\rangle$ to $|2\rangle$, and subsequently from $|2\rangle$ to $|3\rangle$ is comparable to a two-step spontaneous emission; only long after the atom has reached the ground level the fields bring it back to the excited states.

When the Rabi frequency $\Omega_{1}, \Omega_{2}$ are much greater than the decay rates $\gamma_{1}, \gamma_{2}$, the matrix $\mathbf{A}$ is dominated by the coherent part. The transition rate $P_{i \rightarrow j}$ is thus mainly the result of the Rabi oscillations. Here one encounters such a question: what is the difference between the $G_{11}$ and $G_{22}$ functions, provided that the two Rabi frequencies are equal?

As the rigorous analytical solution for the master equation [Eq. (5)] is not possible, we present some numerical results. In Fig. 2 we show the time dependence of $G_{11}(\tau)$ and $G_{22}(\tau)$ for $\gamma_{1}=\gamma_{2}=1, \Omega_{1}=\Omega_{2}=5$. The horizontal axis here is the dimensionless variable $\Omega \pi / 2 \pi$. Comparing the two curves, similarities and differences can be found. The similarity is that both $G_{11}$ and $G_{22}$ oscillate mainly with a frequency of $2 \Omega=2 \sqrt{\Omega_{1}^{2}+\Omega_{2}^{2}}$ but neither $2 \Omega_{1}$ nor $2 \Omega_{2}$. This can be understood in the dressed state picture.

In the results shown in Fig. 2 the decay rates are small compared with the Rabi frequencies; the population transfer probability is mainly determined by the coherent driving fields. For example, when $G_{11}$ is concerned the two dressed states that come from the coupling of field 2 between $|2\rangle$ and $|3\rangle$ can be considered. The Rabi frequency for the transition from level $|1\rangle$ to the two dressed states will be $\sqrt{\Delta^{2}+\Omega_{1}^{2}}$. Here $\Delta$ is the detuning between level $|1\rangle$ and the dressed state, which is just equal to $\Omega_{2}$ in the present case. Therefore the oscillation frequency should be $2 \sqrt{\Omega_{1}^{2}+\Omega_{2}^{2}}$. The same argument is also valid for $G_{22}(\tau)$. In fact, it can be shown that the oscillation frequency for $G_{11}$ and $G_{22}$ is $2 \Omega$, if the decay could be neglected. In the present case, the Rabi frequency is larger than the decay rate, and it is not a bad

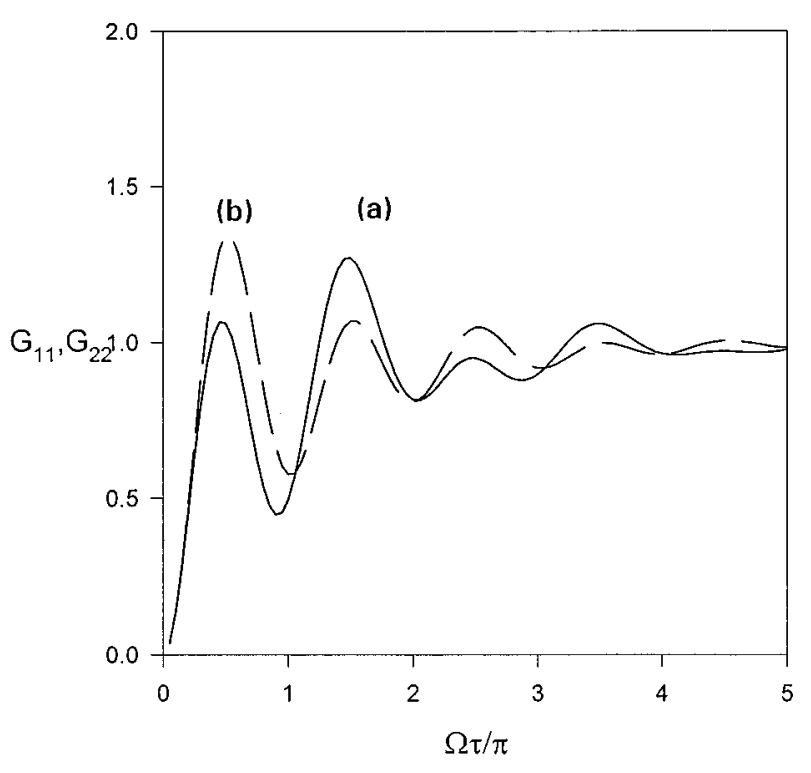

FIG. 2. The $G(\tau)$ factor for the upper transition fluorescence (a), and for the lower transition fluorescence (b) calculated from Eq. (6). The time scale is the inverse of the Rabi oscillation $\sqrt{\Omega_{1}^{2}+\Omega_{2}^{2}}$. Here the parameters are $\gamma_{1}=\gamma_{2}=1, \Omega_{1}=\Omega_{2}=5$.

approximation to neglect the decay. Under this approximation we can find the eigenvalues under the rotating-wave approximation:

$$
\begin{gathered}
\lambda_{0}=0, \quad \lambda_{ \pm}= \pm \Omega, \\
\left|e_{0}\right\rangle=\frac{\Omega_{2}|1\rangle-\Omega_{1}|3\rangle}{\Omega,}, \\
\left|e_{ \pm}\right\rangle=\frac{\Omega_{1}|1\rangle \pm \Omega|2\rangle+\Omega_{2}|3\rangle}{\sqrt{2} \Omega,} .
\end{gathered}
$$

The transition probability from one level to another $P_{i \rightarrow j}$ is predominantly determined by the coherent interaction due to the two lasers. These can be expressed by the probabilities

$$
P_{i \rightarrow j}^{(0)}(\tau)=\left|U_{j i}(\tau)\right|^{2},
$$

where $U$ is the unitary operator representing the evolution of state vector under the coherent interaction

$$
U(t)=\left(\begin{array}{ccc}
H_{0}=\Omega_{1}(|1\rangle\langle 2|+\text { H.c. })+\Omega_{2}(|2\rangle\langle 3|+\text { H.c. }), \\
\frac{\Omega_{1}^{2}}{\Omega^{2}} \cos \Omega t+\frac{\Omega_{2}^{2}}{\Omega^{2}} & -i \frac{\Omega_{1}}{\Omega} \sin \Omega t & -\frac{2 \Omega_{1} \Omega_{2}}{\Omega^{2}} \sin ^{2} \frac{\Omega}{2} t \\
-i \frac{\Omega_{1}}{\Omega} \sin \Omega t & \cos \Omega t & -i \frac{\Omega_{2}}{\Omega} \sin \Omega t \\
-\frac{2 \Omega_{1} \Omega_{2}}{\Omega^{2}} \sin ^{2} \frac{\Omega}{2} t & -i \frac{\Omega_{2}}{\Omega} \sin \Omega t & \frac{\Omega_{2}^{2}}{\Omega^{2}} \cos \Omega t+\frac{\Omega_{1}^{2}}{\Omega^{2}}
\end{array}\right) .
$$




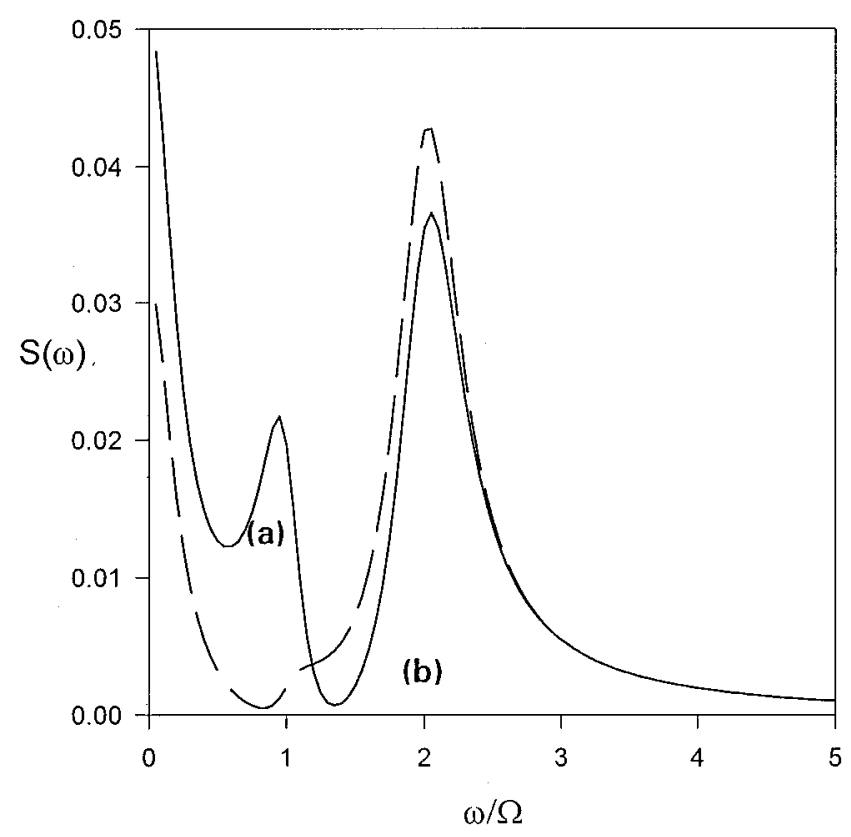

FIG. 3. The spectrum of the intensity correlations defined as in Eq. (9). (a) For upper transition; (b) for lower transition. $\gamma_{1}=\gamma_{2}=1$, $\Omega_{1}=\Omega_{2}=8$.

Therefore we have

$$
\begin{aligned}
& P_{2 \rightarrow 1}^{(0)}=\frac{\Omega_{1}^{2}}{\Omega^{2}} \sin ^{2} \Omega t, \\
& P_{3 \rightarrow 2}^{(0)}=\frac{\Omega_{2}^{2}}{\Omega^{2}} \sin ^{2} \Omega t .
\end{aligned}
$$

While each retains the sinuosodial form of two-level formula, the oscillation frequency for each is no longer determined solely by the corresponding Rabi frequency, $\Omega_{1}$ or $\Omega_{2}$, but by $\Omega$.

Now let us consider the difference between $G_{11}$ and $G_{22}$, which is clearly shown in Fig. 2. The first and third peaks for $G_{11}$ are lower, and the second and fourth peaks are higher than those for $G_{22}$, which indicates that there is another oscillation frequency component in addition to the oscillation at frequency $2 \Omega$ discussed earlier. This is more clearly shown in the spectra of the $G$ functions, defined as

$$
\begin{aligned}
S_{i i}(\omega) & =\left|\int_{0}^{\infty} d t e^{-i \omega t} G_{i i}(t)\right|^{2}, \quad i=1,2, \\
& \sim\left|\tilde{G}_{i i}(z)-\frac{1}{z}\right|_{z=i \omega}^{2} .
\end{aligned}
$$

Here $\tilde{G}$ is the Laplace transform of $G$, and we have removed the singularity at $z=0$ due to the asympototic value of unity. In Fig. 3 the spectra of $G_{11}$ and $G_{22}$ are plotted with the frequency unit of $\Omega$. $\Omega_{1}=\Omega_{2}=5, \gamma_{1}=\gamma_{2}=1$. As shown above the oscillation frequency would been $2 \Omega$ for both $G_{11}$ and $G_{22}$ if the decay could be neglected. Therefore, difference should come from the decay. More clearly it is $\gamma_{2}$ for $G_{11}$ and $\gamma_{1}$ for $G_{22}$. In Fig. 4 we show the spectrum of $G_{11}, G_{22}$ with $\Omega_{1}=\Omega_{2}=8$, and the same decay rates as in Fig. 3. The

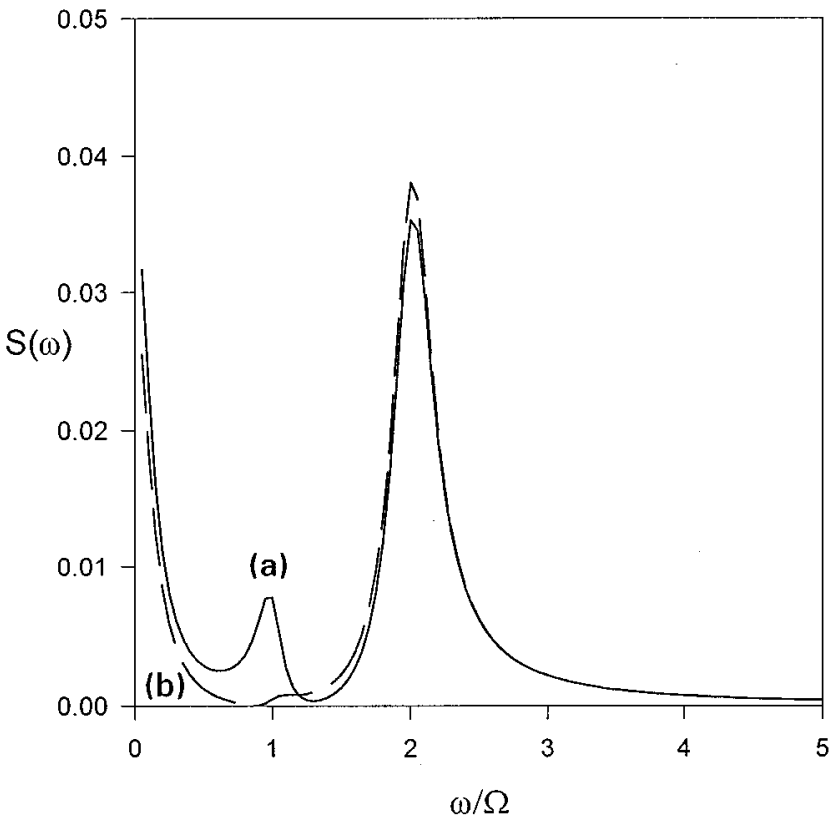

FIG. 4. Similar to Fig. 3, with $\gamma_{1}=\gamma_{2}=1, \Omega_{1}=\Omega_{2}=8$. (a) $G_{11}$; (b) $G_{22}$.

peak at $\Omega$ is lowered in Fig. 4 compared with Fig. 3 as the result of a smaller ratio between the decay rates and the Rabi frequency.

The intensity correlation $G_{i i}$ is the probability of the second spontaneous emission from the level $|i\rangle$, after the first spontaneous emission from the same level. In order to make the second spontaneous emission, the atom must be back to level $|i\rangle$. We consider an atom that has just emitted a photon at frequency $\omega_{23}$ at $t=0$. Immediately after the photon emission it is in the level $i=2$. The probability of bringing the atom back to level $|1\rangle$ by the first driving field is $P_{2 \rightarrow 1}$ in Eq. (16). This is a periodic function with the first maximum at $\Omega t=\pi / 2$. On the other hand, there is a second channel to repopulate level $|1\rangle$. The population in $|2\rangle$ can decay into the level $|3\rangle$ below. The probability of this population decay is proportional to the population in $|2\rangle$, so the first peak is reduced in height. However, once the incoherent process brings the population to $|3\rangle$, it can be driven up to $|1\rangle$ through a two-photon process represented by $P_{3 \rightarrow 1}$, which has a maximum at $\Omega t=\pi$ and the frequency of this process is half of the frequency of $P_{2 \rightarrow 1}$. This delayed transfer back to $|1\rangle$ makes the second peak of $G_{11}$ higher than the first one. The other oscillation periods can be similarly discussed. Thus due to this decay from $|2\rangle$ to $|3\rangle$ followed by the process $P_{3 \rightarrow 1}$, $S_{11}(\omega)$ has another major component at frequency $\Omega$ besides the one at the frequency $2 \Omega$, as shown by curve $a$ in Figs. 3 and 4.

For oscillations in $G_{22}$ we consider the population transfer from $|3\rangle$ to $|2\rangle$ by the absorption of a photon of the second field. This is represented by $P_{3 \rightarrow 2}$, which also has the first maximum at $\Omega t=\pi / 2$. There is no second channel by decay as in the $G_{11}$ case. Therefore there is only one major frequency component present in $S_{22}(\omega)$.

One might argue that in either case, before the the instant of first peak, some population is removed from the original level and distributed to the other two, so all possible decays 


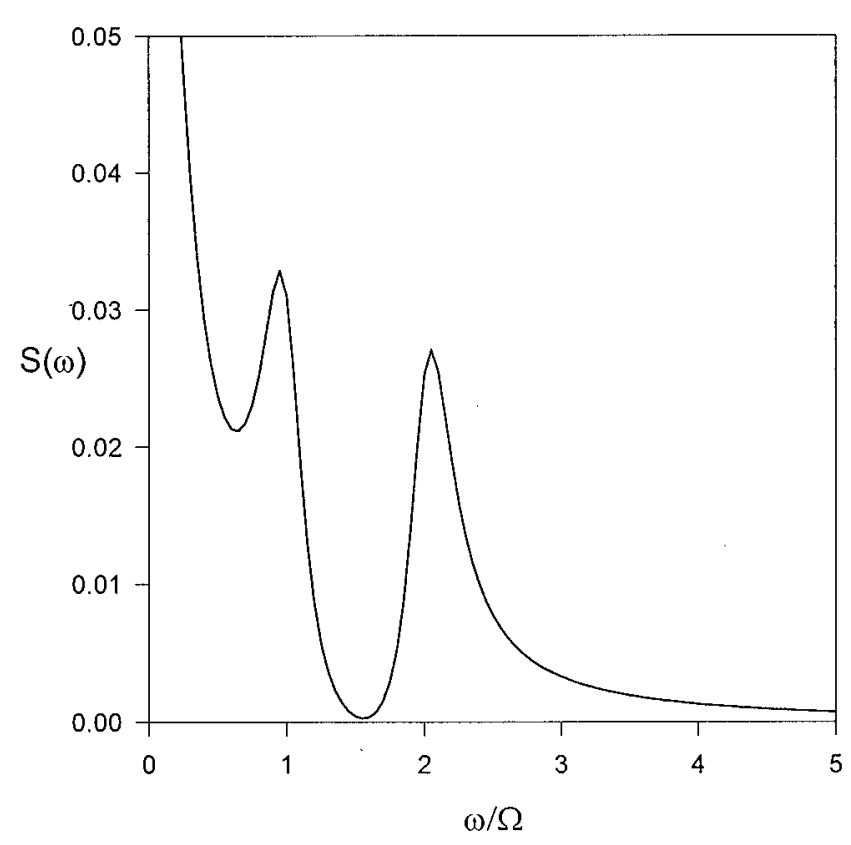

FIG. 5. Spectrum of $G_{11} \cdot \gamma_{1}=\gamma_{2}=1, \Omega_{1}=1, \Omega_{2}=8$.

should be considered. It is true that in the $G_{11}$ case we have decay back to level $|2\rangle$, and in the $G_{22}$ case from $|2\rangle$ back to $|3\rangle$, but these are clearly similar as their effects are proportional to $P_{2 \rightarrow 1}$ and $P_{3 \rightarrow 2}$, respectively, and do not introduce new major frequencies. The decay from $|1\rangle$ back to $|2\rangle$ following a $|3\rangle \rightarrow|1\rangle$ transfer depends on the population in level $|1\rangle$; since level $|1\rangle$ receives population only after level $|2\rangle$ it does not have impact on the peak of level $|2\rangle$ 's population as the decay rate $\gamma_{2}$ on $G_{11}$. Therefore there is a asymmetry between the $|2\rangle \rightarrow|1\rangle$ decay in $G_{11}$, and the $|1\rangle \rightarrow|2\rangle$ decay in $G_{22}$.

To further illustrate our argument, in Fig. 5 we show the spectrum of $G_{11}$ for $\Omega_{1}=1, \Omega_{2}=8$. In this case the second channel is significant due to a large coupling between $|2\rangle$ and $|3\rangle$, which greatly reduces the probability of atoms going back from level $|2\rangle$ to level $|1\rangle$ directly, and greatly increases the probability of going back indirectly by decaying to $|3\rangle$ first, followed by a $P_{3 \rightarrow 1}$ process. Consequently the frequency component at $\omega=\Omega$ becomes larger than the frequency component at $\omega=2 \Omega$ as shown in Fig. 5.

\section{CONCLUSION}

We have shown that the fluorescence intensity correlations from a cascade three-level atom can have secondary oscillations in addition to those due to the Rabi oscillation at the frequency $2 \sqrt{\Omega_{1}^{2}+\Omega_{2}^{2}}$. The behavior differs between the upper and lower transitions, which is counterintuitive. This seemingly contradictory difference can be explained after an analysis of possible channels of decay under the influence of both driving fields.

\section{ACKNOWLEDGMENTS}

The authors (H. H. and M. S. Z.) thank the Hong Kong Baptist University for its hospitality. This work was supported by a RGC grant from the Hong Kong Government. The research of M.S.Z. is supported by the Pakistan Atomic Energy Commission.
[1] G. Alzetta, A. Gozzini, L. Moi, and G. Orriols, Nuovo Cimento 36B, 5 (1976); R. M. Whitley and C. R. Stroud, Jr., Phys. Rev. A 14, 1498 (1976); B. Dalton and P. L. Knight, Opt. Commun. 42, 411 (1982).

[2] M. O. Scully, Phys. Rev. Lett. 55, 2802 (1985); M. O. Scully and M. S. Zubairy, Phys. Rev. A 35, 752 (1987).

[3] S. Harris, Phys. Rev. Lett. 62, 1033 (1989); M. O. Scully, S.-Y. Zhu, and H. Fearn, Z. Phys. D 22, 471 (1992).

[4] L. M. Narducci, G. L. Oppo, and M. O. Scully, Opt. Commun. 75, 111 (1990).

[5] Yifu Zhu, Phys. Rev. A 43, 1502 (1991).

[6] M. Kolwas and P. Lange, Phys. Rev. A 40, 4404 (1990).

[7] P. E. Coleman and P. L. Knight, J. Phys. B 14, 2139 (1981); P. L. Knight, ibid. 12, 3297 (1979); 13, 4551 (1980).
[8] S.-Y. Zhu and M. O. Scully, Phys. Lett. A 201, 85 (1995); A. H. Toor, S.-Y. Zhu, and M. S. Zubairy, Phys. Rev. A 52, 4803 (1995).

[9] J. Kimble and L. Mandel, Phys. Rev. A 13, 2123 (1978); L. Mandel, Opt. Commun. 36, 163 (1981); B. R. Mollow, Phys. Rev. A 188, 1969 (1969).

[10] A. Joshi and R. R. Puri, Phys. Rev. A 43, 6428 (1991); R. D'Souza, A. S. Jayarao, and S. V. Lawande, Phys. Rev. A 41, 4083 (1990).

[11] R. H. Lehmberg, Phys. Rev. A 2, 889, (1970); M. Lax, Phys. Rev. 172, 350 (1968).

[12] A. S. Manka, H. M. Doss, L. M. Narducci, P. Ru, and G. L. Oppo, Phys. Rev. A 43, 3748 (1991); L. M. Narducci, M. O. Scully, G. L. Oppo, P. Ru, and J. R. Tredicce, ibid. 42, 1630 (1990). 